Y. C. Minh and E. F. van Dishoeck, eds.

\title{
Low Temperature Experiments on Gas-Phase Chemical Processes
}

\author{
B. R. Rowe, C. Rebrion Rowe, and A. Canosa \\ Astrochimie Expérimentale, Physique des Atomes, Lasers, Molécules et \\ Surfaces, UMR 6627 du CNRS, Université de Rennes 1, Campus de \\ Beaulieu, 35042 Rennes Cedex, France
}

\begin{abstract}
A review of the most recent experimental results concerning reaction kinetics at low temperatures is presented, most of them having been obtained using the CRESU technique. Some astrochemical consequences are also highlighted.
\end{abstract}

\section{Introduction}

Understanding the evolution of matter all the way from molecular clouds to planetary systems is a key problem for modern astrophysics and astrochemistry. Very often this evolution takes place under cold or ultra-cold conditions such as in interstellar clouds, the outer parts of circumstellar disks, comets and in several planetary atmospheres where a very rich variety of molecules is observed. In order to link observations with chemical modelling, it is essential to gather laboratory data on basic processes over a wide temperature range (typically down to $10 \mathrm{~K}$ ). This papers deals with experimental results obtained below 80 $\mathrm{K}$. The results will be sorted according to their class of reaction, and in each case the state of the art will be presented, together with the latest and key results.

\section{Ion-Molecule Reactions}

Ion-molecule reactions play a central role in the chemistry of interstellar clouds. For technical reasons, they were also the first whose kinetics were studied down to very low temperatures. Ions can be easily stored and manipulated by electromagnetic fields and are detected with very high efficiency. Some techniques, including cryogenic traps (Barlow et al. 1986; Gerlich \& Horning 1992) and supersonic expansion reactors (Rowe et al. 1984; Hawley et al. 1990), operating below $80 \mathrm{~K}$ and yielding rate coefficients and branching ratios directly were developed in the early 1980's. Measurements of cross sections at very low centre-of-mass kinetic energies using merged beams techniques are also available (Gerlich 1989).

Concerning the cryogenically cooled experiments, with the exception of a few measurements performed using drift tube techniques in the early eighties (Böhringer \& Arnold 1983), most of the results relevant to interstellar chemistry have been obtained using ion-trap techniques (Barlow et al. 1986; Gerlich \& Horning 1992). A special advantage of the ion trap is that the ions are stored 
for a long time under very low pressure conditions, and therefore the study of radiative association is possible (Gerlich \& Horning 1992). On the other hand, a serious drawback of cryogenic cooling is that this method restricts the choice of neutral molecules to molecular hydrogen since at the lowest temperatures, all other neutral species freeze out onto the walls.

To overcome this problem, a second class of apparatus has been designed which relies on supersonic expansion to cool down a flow of gas which can consist of either a buffer gas (usually helium) or the neutral reactant itself. This is the principle of the CRESU (Cinétique de Réaction en Ecoulement Supersonique Uniforme) method. Continuous uniform supersonic jets are obtained at low pressure in various buffer gases (usually helium for ion-molecule reaction studies) using suitably contoured Laval nozzles (Dupeyrat et al. 1985) and are used as flow reactors (Rowe et al. 1984). The homogeneous nucleation of the neutral reactant can be avoided, even in supersaturated conditions, by keeping its density sufficiently low. In the most widely used version of this apparatus, a $10 \mathrm{keV}$ electron beam crosses the flow at right angles and a suitable precursor neutral is injected, allowing the formation of the reactant ions. By monitoring the reactant and product ions as a function of neutral reactant density, using a quadrupole mass spectrometer located downstream of this electron-beam, rate coefficients can be obtained by standard flow reactor analysis. A drawback of the technique is that neutral precursor and reactant have to be mixed together with the buffer gas in the nozzle reservoir. This makes it difficult to derive branching ratios. To overcome this problem, a selective ion injection method was developed by Rowe et al. (1989). A new system is currently under development in our laboratory.

The free jet flow reactor of Mark Smith and co-workers (Hawley et al. 1990) is in principle very similar to the CRESU apparatus. In this technique, a free jet expansion is used in place of the uniform flow. The ions are formed by the REMPI (Resonant Enhanced Multiphoton Ionisation) of a suitable precursor and are detected by a time of flight mass spectrometer. The advantages of this technique are that the ions can be formed in well defined states by REMPI and that a temperature close to $0 \mathrm{~K}$ can be achieved. A serious drawback however, is that thermodynamic conditions are not so well defined as in an uniform flow. The velocity distribution of ions and neutrals is no longer Maxwell-Boltzmann and so two temperatures (perpendicular and parallel) have been defined. The rotational temperature is also usually much higher than the kinetic temperature.

Instead of measuring thermal rate coefficients directly, it is also possible to design an experiment to obtain a cross section $\sigma$ as a function of the relative kinetic energy in the centre of mass $E=1 / 2 \mu v^{2}$ ( $\mu$ reduced mass, $v$ relative velocity of the collision partner). The rate coefficient can then be obtained by:

$$
k(T)=\sum_{i, j} x_{A_{i}} x_{B_{j}} \int_{0}^{\infty} 8 \pi \frac{\mu E}{(2 \pi \mu k T)^{3 / 2}} \sigma_{i, j}(E) e^{-E / k T} d E
$$

The subscripts $i$ and $j$ refer to the internal state of the reactants $\mathrm{A}$ and $\mathrm{B}$ for which the molar fraction in state $i, j$ is represented by the terms $x_{A_{i}}$ and $x_{B_{j}}$. In order to obtain $k(T)$ at very low $T$, the cross section has to be measured at extremely low values of $E$. This is extremely difficult but can in principle 
be achieved with the merged beam technique where two beams, of ions and of neutral reactant respectively, are made co-linear with a very low relative velocity. Such an apparatus has been built in the group of Gerlich at Chemnitz (Gerlich 1989). At the lowest energy however, the resolution of the merged beam techniques is comparable to the collision energy so that a de-convolution of the results must be made, assuming that the energy distribution is known. The internal state (especially rotation) of the neutral reactant is also not always well specified, in contrast to the case of ion traps where the ions can perhaps relax to their ground state.

In the last twenty years, a large database on ion-molecule reactions has been developed which, together with theoretical work, allows us to derive some trends (see for example the excellent review by Smith 1994). Reactions which are fast at room temperature (i.e. occurring at the capture rate) remain fast at very low temperatures and are well described by capture theories (Rowe et al. 1995). Basically, for an impact parameter below the critical value under which the centrifugal energy barrier does not prevent the collision partners from colliding (assuming no repulsive short range part of the potential) the reaction is assumed to proceed with a $100 \%$ efficiency. Various calculations can be performed, the most famous of them being the very simple Langevin formula derived for a long range ion-induced dipole intermolecular potential which is suitable for non-polar molecules:

$$
k_{L}=2 \pi q \sqrt{\frac{\alpha}{\mu}} \text { using } \quad V(r)=-\frac{\alpha q^{2}}{2 r^{4}}
$$

where $q$ is the ion charge, $\alpha$ is the polarisability of the neutral reactant and $\mu$ is the reduced mass (all in cgs units). This Langevin formula predicts that the rate coefficient is independent of temperature. Table 1 lists a variety of reactions that have been studied. It is clear that the rate coefficient remains very close to the Langevin value down to very low temperatures.

When the neutral reactant is a polar molecule, the long range intermolecular potential is more complicated and takes the form:

$$
V(r)=-\frac{\alpha q^{2}}{2 r^{4}}-\frac{q D}{r^{2}} \cos \theta
$$

where $\theta$ is the angle between the dipole axis and the line joining the centreof-mass of the colliding partners. Various methods have been used to calculate the rate coefficient in this case (Su \& Chesnavich 1982; Clary 1987), some of them leading to an analytical formula for $k(T)$ (Su \& Chesnavich 1982; Troe 1985), the most sophisticated (Troe 1985; Clary 1987) allowing one to predict the kinetic behaviour as a function of the rotational state of the neutral reactant. For temperatures down to $10 \mathrm{~K}$, all these methods are in agreement and reproduce the increase of the rate constant when $T$ decreases, that is displayed by the experimental results. As an example, Figure 1 displays the temperature dependence of the rate coefficient for the reaction $\mathrm{N}^{+}+\mathrm{NH}_{3}$.

As a general rule of thumb it can be considered that reactions which are fast at room temperature remain fast at very low temperature, and the dependence of $k$ on $T$ depends on the dipole moment of the neutral. In many cases, however, slow reactions display a reverse temperature dependence, and their rate coeffi- 
Table 1. Rate coefficients obtained for non-polar molecules. Numbers are expressed in $10^{-9} \mathrm{~cm}^{3} \mathrm{~s}^{-1}$ units. Results at $0.3 \mathrm{~K}$ were obtained in a free jet apparatus (Smith 1994) whereas others were obtained using the CRESU technique.

\begin{tabular}{c|cccccc}
\hline Reactants & $0.3 \mathrm{~K}$ & $8 \mathrm{~K}$ & $20 \mathrm{~K}$ & $23 \mathrm{~K}$ & $30 \mathrm{~K}$ & $\mathrm{k}_{L}$ \\
\hline $\mathrm{He}^{+}+\mathrm{N}_{2}$ & & 1.2 & 1.3 & 1.6 & 1.3 & 1.7 \\
$\mathrm{He}^{+}+\mathrm{O}_{2}$ & & 1.0 & 0.85 & & & 1.6 \\
$\mathrm{He}^{+}+\mathrm{CO}$ & & 1.5 & 1.4 & & & 1.8 \\
$\mathrm{~N}^{+}+\mathrm{O}_{2}$ & & 0.55 & & & & 0.95 \\
$\mathrm{~N}^{+}+\mathrm{CH}_{4}$ & 0.82 & & & & 1.4 \\
$\mathrm{~N}^{+}+\mathrm{CO}$ & & 1.1 & & & 1.7 & 1.1 \\
$\mathrm{H}_{3}^{+}+\mathrm{CO}$ & & & & & 1.3 & 1.9 \\
$\mathrm{H}_{3}^{+}+\mathrm{N}_{2}$ & & & & & 1.9 & 2.4 \\
$\mathrm{H}_{3}^{+}+\mathrm{CH}_{4}$ & & & & 0.9 & & 1.0 \\
$\mathrm{C}^{+}+\mathrm{O}_{2}$ & 2.4 & & & & & 1.4 \\
$\mathrm{C}^{+}+\mathrm{CH}_{4}$ & 1.4 & & & & & 1.5 \\
$\mathrm{C}^{+}+\mathrm{N}_{2} \mathrm{O}$ & 1.8 & & & & & \\
\hline
\end{tabular}

cients increase toward the capture rate at very low temperatures. A striking example (although not relevant to interstellar chemistry) is the $\mathrm{Cl}^{-}+\mathrm{CH}_{3} \mathrm{Br}$ reaction. The temperature dependence of this rate coefficient is shown in Figure 2 and is well represented by a $\mathrm{S}_{N} 2$ mechanism (Le Garrec et al. 1997b). Unfortunately this kind of behaviour cannot be generalised especially for the case of slow reactions with molecular hydrogen (e.g. the $\mathrm{He}^{+}+\mathrm{H}_{2}$ reaction which remains very slow at very low temperature).

Several uncertainties remain however, with regard to the behaviour of some classes of ion-molecule reactions under interstellar conditions. Almost no data are available for reactions between ions and free radicals and there is a scarcity of data concerning radiative associations, most of the latter having been obtained from trap experiments. It would also be of interest to have more information concerning branching ratios, especially for large molecules, and their possible variation with temperature.

\section{Radical-Neutral Reactions}

Many of the techniques developed for ion-molecule studies, especially the cryogenic ones, are unfortunately not suitable for uncharged species since they rely on the use of electromagnetic fields to manipulate, store and collect the charged reactants. The first measurement of reaction rates of neutral radicals with molecules down to temperatures of $13 \mathrm{~K}$ was performed seven years ago. This is the result of a very fruitful collaboration between our group and the group of I.W.M. Smith at the School of Chemistry at Birmingham (UK), using the PLPLIF (Pulsed Laser Photolysis - Laser Induced Fluorescence) method together 


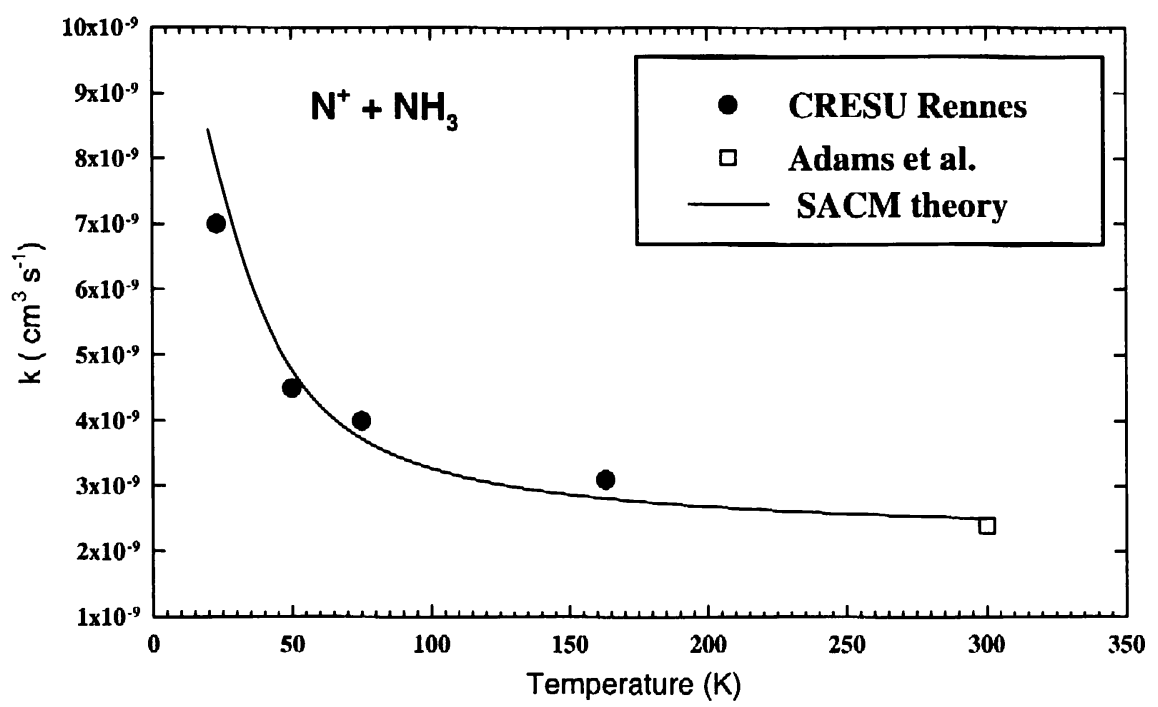

Figure 1. Rate coefficient for the reaction $\mathrm{N}^{+}+\mathrm{NH}_{3}$ as a function of temperature. Experimental results (Rowe et al. 1995; Adams et al. 1980) are compared to a theoretical model (Troe 1985).

with the CRESU technique (Sims et al. 1992, 1994). Two continuous flow apparatuses exist now for neutral-neutral reaction studies, one in Rennes, the other in Birmingham (Chastaing et al. 1998), and a pulsed version has been built by M. Smith in Tucson, Arizona (Atkinson \& Smith 1995). A sketch of the Rennes experiment which has been described in detail elsewhere (Sims et al. 1994), is shown in Figure 3. As for ion-molecule reactions, the uniform supersonic flow at the exit of a Laval nozzle is used as a reactor cell. In the reservoir of the nozzle, a radical precursor is mixed together with a reactant neutral and with the buffer gas. The reservoir and the nozzle are made moveable with respect to the detection system, which consists of a number of optics and of a photomultiplier. The whole experiment is set in a vacuum chamber pumped by large Roots blowers $\left(24000 \mathrm{~m}^{3} / \mathrm{h}\right)$. Two pulsed lasers beams are made co-linear on the nozzle axis. The first laser forms the reactant radical by photolysis of the suitable precursor. This radical is detected by LIF (Laser Induced fluorescence) using the second (tuneable) laser and its temporal behaviour for various densities of neutral reactant, is analysed. Standard techniques then allow one to derive the rate coefficient (Sims et al. 1994; Le Picard et al. in prep.). Reactions of molecular radicals $\left(\mathrm{CN}, \mathrm{CH}, \mathrm{OH}, \mathrm{C}_{2} \mathrm{H}\right)$ or atoms (including carbon atoms and metallic species: $\mathrm{Al}$ and $\mathrm{Si}$ ) with stable species have now been studied. The experiments performed at Birmingham with carbon atoms (Chastaing et al. 1999) and the $\mathrm{C}_{2} \mathrm{H}$ radical (Chastaing et al. 1998) relied on chemi-luminescence detection instead of LIF, but experiments using VUV (vacuum ultraviolet) LIF have started for carbon atoms. All these radicals exhibit large reaction rate 


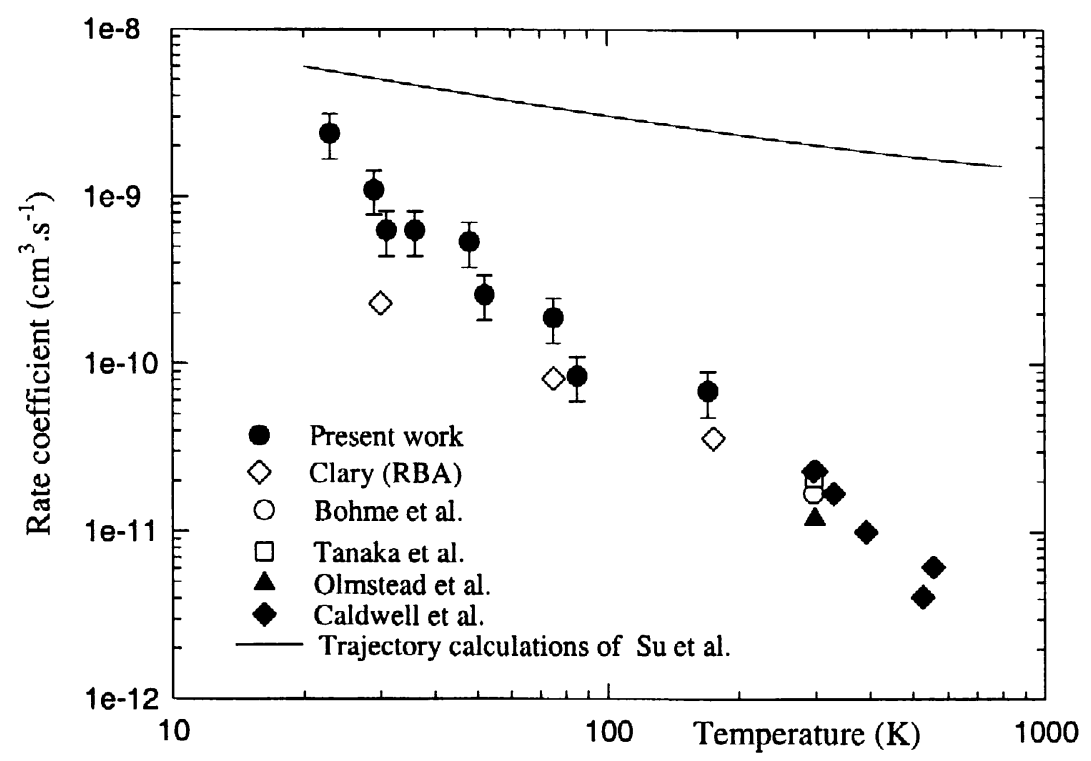

Figure 2. Rate coefficient for the reaction $\mathrm{Cl}^{-}+\mathrm{CH}_{3} \mathrm{Br}$ as a function of temperature (Le Garrec et al. 1997b and references therein).

coefficients (close to the collisional limit) at very low temperatures, displaying occasionally a strong reverse temperature dependence below room temperature.

Table 2 lists the rate coefficients of some reactions for a given temperature. A first glance at this table shows that reactions which are fast at room temperature (i.e. occurring at a rate close to the collisional value of a few $10^{-10} \mathrm{~cm}^{3} \mathrm{~s}^{-1}$ molecule ${ }^{-1}$ ) remain fast at low temperatures exhibiting sometimes an inverse temperature dependence.

In fact a rich variety of behaviour can be noticed: reactions which are slower at room temperature can exhibit a strong inverse temperature dependence such as $\mathrm{CN}+\mathrm{O}_{2}$ or $\mathrm{CN}+\mathrm{NH}_{3}$ (Sims et al. 1994). A special case is the reaction of the $\mathrm{CN}$ radical with ethane where the rate coefficient first decreases with temperature and then increases strongly, a totally unexpected behaviour (Sims et al. 1993). Sometime the rate coefficient reaches a shallow maximum at temperatures around $20-80 \mathrm{~K}$ and then decreases as shown in Figure 4 for the $\mathrm{Si}+\mathrm{O}_{2}$ and $\mathrm{C}_{2} \mathrm{H}_{4}$ reactions (Le Picard et al. in prep.; Gougeon et al. in prep.). Such a variety of behaviour is not well reproduced at the present time by capture theory even if in some cases a fairly good agreement can be achieved as shown in Figure 5 for the $\mathrm{Al}+\mathrm{O}_{2}$ reaction (Reignier et al. 1998).

A major problem is that collision theory is not yet sufficiently advanced to make reliable predictions. This can be due to the lack of accurate intermolecular potentials and to the fact that for neutral-neutral interactions, the distinction between long and short range forces is not so well established as for ions. Many 


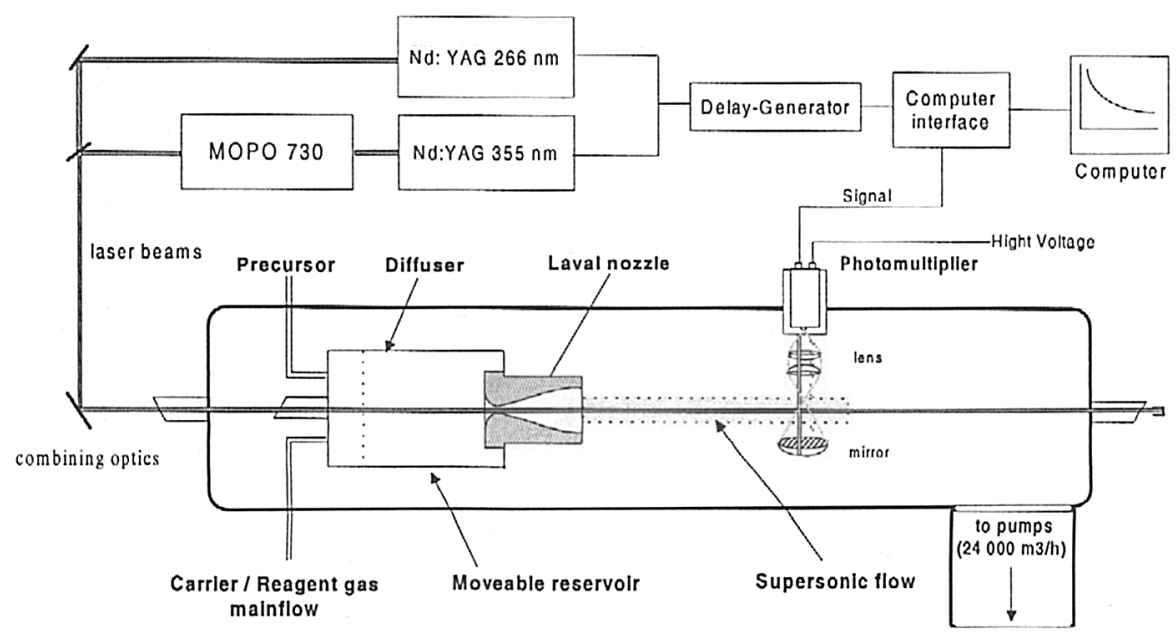

Figure 3. Sketch of the CRESU chamber used for the study of neutral neutral reactions.

of the reactions included in Table 2 can have a strong influence on the chemistry of the interstellar medium as discussed by Herbst et al. (1994) and Chastaing et al. (1998). From such results it has become clear that the effects of neutral chemistry have been strongly underestimated in the modelling of interstellar clouds. This important role for neutral chemistry has been overlooked in the past and a considerable amount of work clearly remains to be done in this field.

\section{Relaxation Processes}

The excitation and relaxation of spin-orbit and rotational states play a key role in astrochemistry since they control the radiation that is emitted by the interstellar medium in a wavelength range of particular interest. These processes influence therefore, the interpretation of radioastronomical observations. They are also directly linked to the thermal balance of interstellar clouds, the energy being able to escape through radiative spontaneous emission following excitation (Dyson \& Williams 1997). In fact very few experimental results are available concerning these processes in the relevant temperature range. Most have been obtained very recently in fact using the CRESU technique. In the case of the spin-orbit state, the PLP-LIF technique can be used to measure the rate coefficient for the relaxation process. The technique does not differ from that used for chemical reactions and requires only that the distribution of spin-orbit states created by photolysis is not thermal and that a suitable LIF spectrum exists for the atom or molecule studied.

As an example, Figure 6 shows the LIF spectrum of aluminium atoms for different delays between the photolysis and LIF laser pulses. Analysis of the 


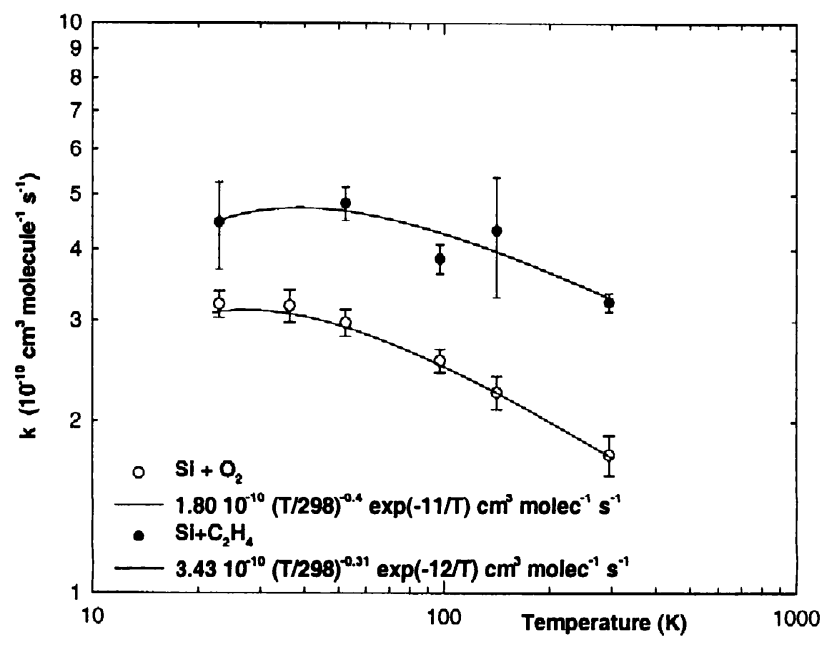

Figure 4. Rate coefficients for the reactions $\mathrm{Si}+\mathrm{O}_{2}$ and $\mathrm{Si}+\mathrm{C}_{2} \mathrm{H}_{4}$ as a function of temperature.

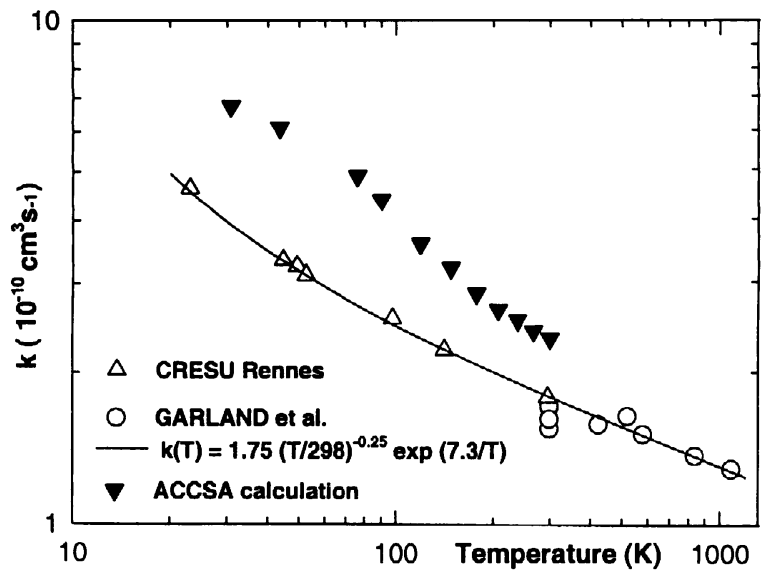

Figure 5. Rate coefficients of the reaction $\mathrm{Al}+\mathrm{O}_{2}$ as a function of temperature (Le Picard et al. 1997 and references therein). 
Table 2. Rate coefficients obtained for different neutral-neutral reactions. Numbers are expressed in $10^{-10} \mathrm{~cm}^{3} \mathrm{~s}^{-1}$ units and correspond to the minimum temperature of experiments $(\mathrm{a}: 13 \mathrm{~K}, \mathrm{~b}: 15 \mathrm{~K}, \mathrm{c}: 23 \mathrm{~K}, \mathrm{~d}: 25$ $\mathrm{K}$, and e:53 K). Numbers in italic correspond to association reactions. $\mathrm{C}_{2} \mathrm{H}$ and carbon atoms were studied at the University of Birmingham either by chemi-luminescence techniques $\left(\mathrm{C}_{2} \mathrm{H}, \mathrm{C}\right)$ or VUV techniques (C(UV), Chastaing et al. in prep.).

\begin{tabular}{|c|c|c|c|c|c|c|c|c|c|}
\hline & | & & & & ical & & & & \\
\hline Reactant & $1 \mathrm{CN}$ & $\mathrm{OH}$ & $\mathrm{CH}(\mathrm{v}=0)$ & $\mathrm{CH}(\mathrm{v}=1)$ & $\mathrm{Al}$ & $\mathrm{Si}$ & $\mathrm{C}_{2} \mathrm{H}$ & $\mathrm{C}$ & $\mathrm{C}(\mathrm{UV})$ \\
\hline $\mathrm{O}_{2}$ & $1.34^{a}$ & & $1.54^{a}$ & & $4.63^{c}$ & $3.21^{\mathrm{c}}$ & $1.05^{b}$ & $1.19^{b}$ & $1.12^{b}$ \\
\hline $\mathrm{H}_{2}$ & & & $0.03 \mathscr{Z}^{e}$ & $1.87^{c}$ & & & & & \\
\hline $\mathrm{D}_{2}$ & & & $1.43^{a}$ & $1.93^{c}$ & & & & & \\
\hline $\mathrm{N}_{2}$ & & & $0.004^{e}$ & $1.11^{c}$ & & & & & \\
\hline NO & & $0.035^{c}$ & $2.34^{a}$ & & & & & & $2.28^{b}$ \\
\hline $\mathrm{CO}$ & & & $0.061^{e}$ & $1.55^{c}$ & & & & & \\
\hline $\mathrm{HBr}$ & & $1.07^{c}$ & & & & & & & \\
\hline $\mathrm{NH}_{3}$ & $4.09^{d}$ & & $2.21^{\mathrm{c}}$ & & & & & & \\
\hline $\mathrm{CH}_{4}$ & & & $3.16^{c}$ & & & & & & \\
\hline $\mathrm{C}_{2} \mathrm{H}_{2}$ & $4.60^{d}$ & & $3.85^{c}$ & & & & $2.27^{b}$ & $3.46^{b}$ & $4.76^{b}$ \\
\hline $\mathrm{C}_{2} \mathrm{H}_{4}$ & $4.35^{d}$ & & $3.92^{c}$ & & & $4.47^{c}$ & $2.17^{b}$ & $3.40^{b}$ & $4.79^{b}$ \\
\hline $\mathrm{C}_{2} \mathrm{H}_{6}$ & $1.13^{d}$ & & $2.47^{c}$ & & & . & & & \\
\hline $\mathrm{C}_{3} \mathrm{H}_{6}$ & & & & & & & $1.14^{b}$ & $2.88^{b}$ & \\
\hline Z-but-2-ene & & $3.89^{c}$ & & & & & & & \\
\hline E-but-2-ene & & $4.52^{c}$ & & & & & & & \\
\hline but-1-ene & & $4.2 x$ & $3.95^{c}$ & & & & & & \\
\hline
\end{tabular}

decrease of the signal for the upper state allows one to obtain the rate coefficient (Le Picard et al. 1998). Preliminary results have recently been obtained both at Rennes and Birmingham respectively for silicon and carbon atoms.

The study of rotational relaxation requires the development of a new technique. At Birmingham, the IRUVDR (Infra Red Ultra Violet Double Resonance) has been implemented in the CRESU experiment. A tuneable IR pump laser populates selected ro-vibrational levels of a given molecule. A second tuneable UV probe laser detects a specific state by LIF. To date the technique has allowed the study of the rotational relaxation of $\mathrm{NO}$ by $\mathrm{He}$ and the vibrational relaxation of NO by NO to be made, down to $7 \mathrm{~K}$ (James et al. 1998). The study of systems more relevant to the interstellar medium is planned.

\section{Electron Attachment}

If the idea that negative charges could be carried at least partly by anions in interstellar clouds is correct, the physics and chemistry of these clouds would be deeply modified. The ionic route leading to the formation of molecules would be drastically changed and as the mobility of anions is much less than the mobility of electrons, this will strongly influence models of gravitational collapse. The possible assignment of some DIBs (Diffuse Interstellar Bands) to $\mathrm{C}_{n}^{-}$anions (Kirwood et al. 1998) also raises the question of their formation, and electron attachment is a possible route. 

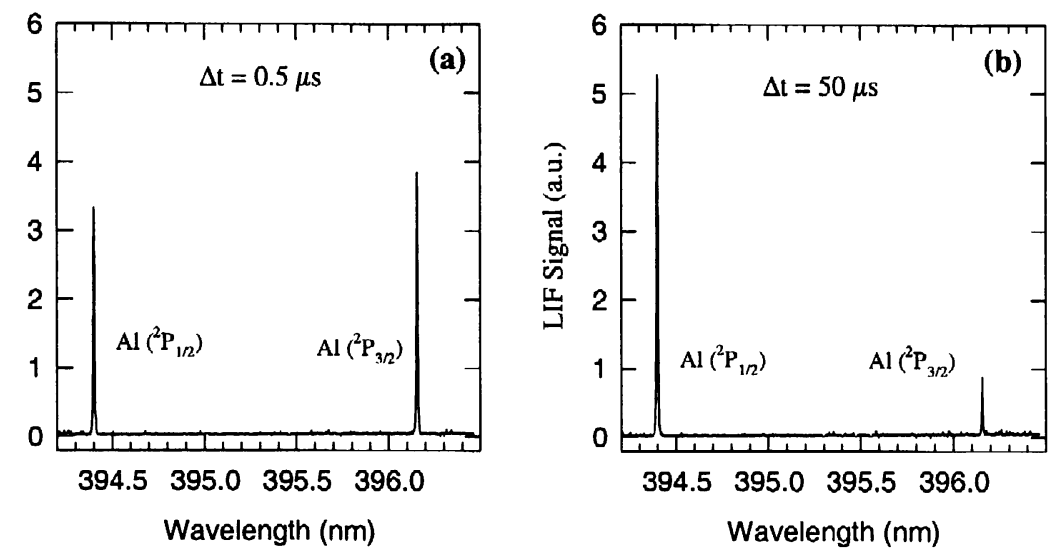

Figure 6. LIF spectra of the $\left({ }^{2} \mathrm{~S}_{1 / 2}-{ }^{2} \mathrm{P}_{1 / 2}\right)$ and $\left({ }^{2} \mathrm{~S}_{1 / 2}-{ }^{2} \mathrm{P}_{3 / 2}\right)$ lines of $\mathrm{Al}$ recorded at $52.8 \mathrm{~K}$ at two different delay times: (a) before relaxation occurred $(\Delta \mathrm{t}=0.5 \mu \mathrm{s}) ;(\mathrm{b})$ after relaxation occurred $(\Delta \mathrm{t}=50 \mu \mathrm{s})$.

There exist a variety of techniques allowing one to study electron attachment at very low energy. Most are non-thermal methods where cross sections are obtained as a function of electron energy, sometimes with a very high energy resolution (see the review by Dunning 1995). As the electron is very light, the kinetic energy of the electron is basically equal to the relative kinetic energy in the centre-of-mass. It follows that it is straightforward in this case to derive a rate coefficient through the use of formula (1). The principal techniques are the threshold photoelectron technique, where electrons are produced by photoionisation very close to threshold, and the Rydberg atom technique where atoms are produced in a well specified Rydberg state. The Rydberg electron behaves basically as a free electron and electron attachment can be simulated by collisions such as:

$$
\mathrm{A}^{*}+\mathrm{M} \rightarrow \mathrm{A}^{+}+\mathrm{M}^{-}
$$

$\mathrm{A}^{*}$ referring to the Rydberg atoms.

These techniques however, have not been developed for interstellar applications, and the systems studied to date present no real interest for the interstellar medium. In most of these experiments, furthermore, the neutral molecules are kept at room temperature. We have recently applied the CRESU experiment to the study of electron attachment and have demonstrated the very strong influence of the internal state of the neutral molecule on the attachment process (at least for dissociative attachment) (Le Garrec et al. 1997a). This is illustrated in Figure 7 which shows the rate coefficient for the process:

$$
\mathrm{e}+\mathrm{CF}_{3} \mathrm{Br} \rightarrow \mathrm{Br}^{-}+\mathrm{CF}_{3}
$$




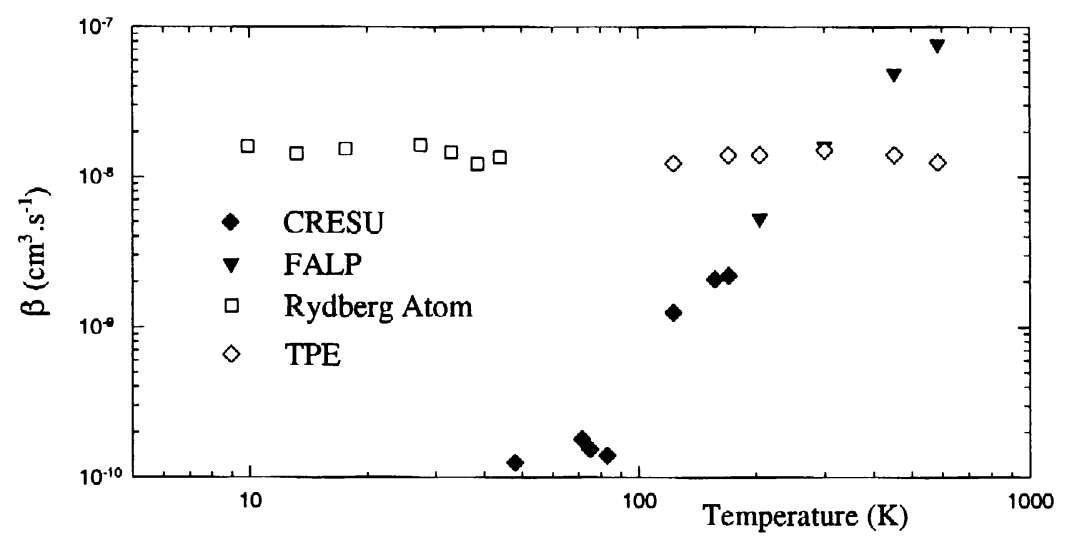

Figure 7. Rate coefficients of the reaction $\mathrm{e}+\mathrm{CF}_{3} \mathrm{Br}$ as a function of temperature (TPE $=$ Threshold Photo Electrons).

as a function of temperature. The dramatic difference in behaviour observed between the FALP and CRESU experiments and the other techniques is due to the vibrational population of $\mathrm{CF}_{3} \mathrm{Br}$ increasing with increasing gas temperature. In the Rydberg atom and threshold photoionisation experiments, the gas was held at $300 \mathrm{~K}$ while the electron energy was varied. We have also studied electron attachment to anthracene $\left(\mathrm{C}_{14} \mathrm{H}_{10}\right)$, one of the simplest Polycyclic Aromatic Hydrocarbons, down to $50 \mathrm{~K}$ (Mostefaoui et al. 1998). These measurements show no significant variation of the rate coefficient which remains close to the room temperature value of $1.0 \times 10^{-9} \mathrm{~cm}^{3} \mathrm{~s}^{-1}$. Although no pressure dependence of this bimolecular coefficient was found in the experiment, this result refers to a collisionally stabilised process. For interstellar applications, it shows that there is no activation energy barrier but a knowledge of the radiative rate is required. It could be inferred from our data if the lifetime (for autodetachment) of the excited transient anion was known.

\section{Neutral Dimer Formation}

Molecules bonded by Van der Waals forces could be of great astrochemical interest (Allan et al. 1997). Although the spectroscopy of neutral dimers has been extensively studied, however, very little is known about their process of formation. Recently, using the CRESU apparatus in Rennes we have been able to study the kinetics of benzene dimer formation down to $15 \mathrm{~K}$ (Hamon et al. 2000). As expected, the rate coefficient displays a strong inverse temperature dependence with a bimolecular value approaching a large fraction of the collisional rate at the lowest temperature, as shown in Figure 8. Considering the large 


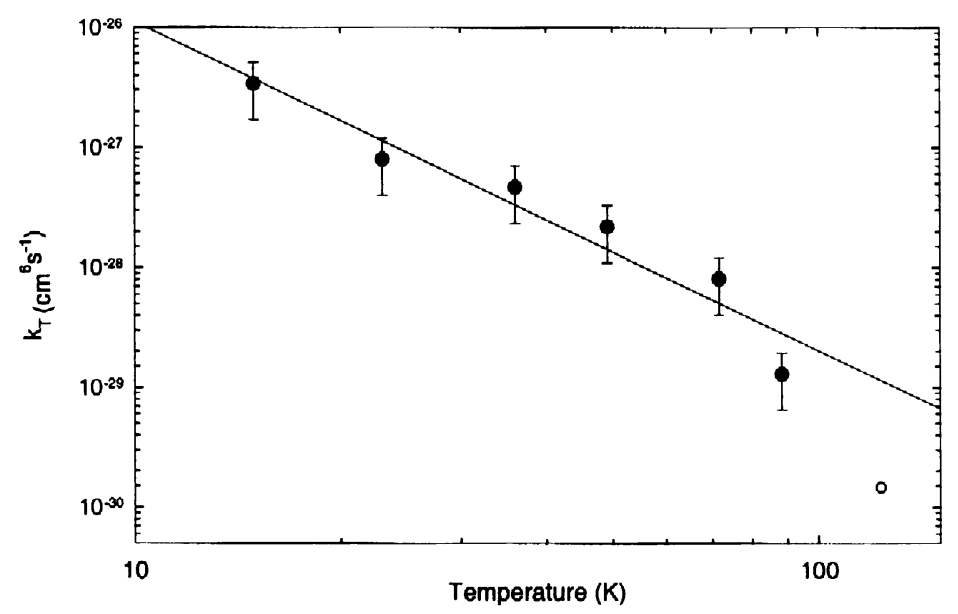

Figure 8. Association rate coefficient for the reaction $\mathrm{C}_{6} \mathrm{H}_{6}+\mathrm{C}_{6} \mathrm{H}_{6}$ + He as a function of temperature.

size of the dimer, therefore, the radiative process is probably fast at very low temperatures. The question of dimer formation in interstellar space probably deserves much more work.

\section{Conclusion}

Ten years ago it would have been easier to make a review of gas phase processes at very low temperatures since most of the results were restricted to ion-molecule reactions. The field has broadened a lot in the last years, however, and a variety of processes are now studied in the relevant temperature range for the interstellar medium. The present review gives therefore, just a flavour of what has been done. What remains to be done is however, an enormous amount of work, especially in the field of the chemistry of complex molecules at low temperatures and only a few research groups are involved in this field. Astrochemistry will probably keep the laboratory study of gas phase processes busy for many years.

Acknowledgments. The authors wish to acknowledge Dr. Ian Sims and Professor Ian Smith of the University of Birmingham, UK for providing some results prior to publication.

\section{References}

Adams, N.G., Smith, D., \& Paulson, J.F. 1980, J. Chem. Phys., 72, 288

Allan, R.J., Loinard, L., Mc Kellar, A.R.W., \& Lequeux, J. 1997, ApJ, 489, 102 Atkinson, D.B. \& Smith, M.A. 1995, Rev. Sci. Instrum., 66, 4434 
Barlow, S.E., Luine, J.A., \& Dunn, G.H. 1986, Int. J. Mass Spectrom. Ion Proc., 74,97

Böhringer, H. \& Arnold, F. 1983, Int. J. Mass Spectrom. Ion Phys., 49, 61

Chastaing, D., James, P.L., Sims, I.R., \& Smith, I.W.M. 1998, Faraday Discuss., 109,165

1999, Phys. Chem. Chem. Phys., I, 224

Chastaing, D., et al. 2000, in preparation

Clary, D.C. 1987, J. Chem. Soc. Faraday Transactions II, 83, 139

Dunning, F.B. 1995, J. Phys. B, 28, 1645

Dupeyrat, G., Marquette, J.B., \& Rowe B.R. 1985, Phys. of Fluids, 28, 1273

Dyson, J.E. \& Williams, D.A. 1997, in The physics of the interstellar medium, graduate series in Astronomy (IOP), 28

Gerlich, D. 1989, in XII Int. Symp. on Molecular Beams, V. Aquilanti (Perugia), 37

Gerlich, D. \& Horning, S. 1992, Chem. Rev., 92, 1509

Gougeon, S., Le Picard, S.D., Canosa, A., Rebrion-Rowe, C., \& Rowe, B.R. 2000 , in preparation

Hamon, S., Le Picard, S.D., Canosa, A., Rowe, B.R., \& Smith, I.W.M. 2000, J. Chem. Phys., submitted

Hawley, M.T., Mazely, T.L., Randeniya, L.K., Smith, R.S., Zeng, X.K., \& Smith, M.A. 1990, Int. J. Mass Spectrom. Ion Proc., 80, 239

Herbst, E., Lee, H.H., Howe, D.A., \& Millar, T.J. 1994, MNRAS, 268, 335

James, P.L., Sims, I.R., Smith, I.W.M., Alexander, M.H., \& Yang, M. 1998, J. Chem. Phys., 109, 3882

Kirwood, D.A., Linnartz, H., Grutter, M., Dopfer, O., Motylewski, T., Pachkov, M., Tulej, M., Wyss, M., \& Maier, J.P. 1998, Faraday Discuss., 109, 109

Le Garrec, J.L., Sidko, O., Queffelec, J.L., Mitchell, J.B.A., \& Rowe, B.R. 1997a, J. Chem. Phys., 107, 54

Le Garrec, J.L., Rowe, B.R., Queffelec, J.L., Mitchell, J.B.A., \& Clary, D.C. 1997b, J. Chem. Phys., 107, 1021

Le Picard, S.D., Bussery-Honvault, B., Rebrion-Rowe, C., Honvault, P., Canosa, A., Launay, J.M., \& Rowe, B.R. 1998, J. Chem. Phys., 108, 10319

Le Picard, S.D., Canosa, A., Chastaing, D., Travers, D., Rowe, B.R., \& Stoecklin T. 1997, J. Phys. Chem. A, 101, 9988

Le Picard, S.D., Canosa, A., Rebrion-Rowe, C., Rowe, B.R., \& Pineau des Forêts, in preparation

Mostefaoui, T., Rebrion-Rowe, C., Le Garrec, J.L., Rowe, B.R., \& Mitchell, J.B.A. 1998, Faraday Discuss., 109, 71

Reignier, D., Stoecklin ,T., Le Picard, S.D., Canosa, A., \& Rowe, B.R. 1998, J. Chem. Soc. Faraday Trans. 94, 1681

Rowe B.R., Canosa A. \& Le Page V. 1995, Int. J. Mass Spectrom. Ion Proc., $149 / 150,573$

Rowe, B.R., Dupeyrat, G., Marquette, J.B., \& Gaucherel, P. 1984, J. Chem. Phys., 80, 4915

Rowe, B.R., Marquette, J.B., \& Rebrion, C. 1989, J. Chem. Soc. Faraday Transactions II, 85, 1631

Sims I.R., Queffelec J.L., Defrance A., Rebrion-Rowe C., Travers D., Bocherel P., Rowe B.R. \& Smith I.W.M. 1994, J. Chem. Phys., 100, 4229

Sims I.R., Queffelec J.L., Defrance A., Rebrion-Rowe C., Travers D., Rowe B.R. \& Smith I.W.M. 1992, J. Chem. Phys., 97, 8798 
Sims I.R., Queffelec J.L., Travers D., Rowe B.R., Herbert L., Karthauser J. \& Smith I.W.M. 1993, Chem. Phys. Lett., 211, 461

Smith M.A. 1994, in Unimolecular and bimolecular ion-molecule reaction dynamics, eds. C.Y. Ng, T. Baer \& I. Powis, 183

$\mathrm{Su}$, T. \& Chesnavich, W.J. 1982, J. Chem. Phys., 76, 5183

Troe, J. 1985, J. Chem. Phys., 122, 425

\section{Discussion}

E. Herbst: (1) Will you be able to measure the products of neutral-neutral reactions? (2) Does the $e-\mathrm{C}_{14} \mathrm{H}_{10}$ reaction possess an activation energy barrier since the rate coefficient is less than s-wave capture?

B. Rowe: (1) We wish to do it in the future and some experiments are also planned by the group of Ian Smith at Birmingham in their CRESU apparatus. (2) The fact that the rate coefficient does not change with temperature indicates no activation energy barrier - we have no explanation for the rate being lower than s-wave capture. A possible explanation would be that elections are not thermalized in the CRESU experiment. However we have made calculations and experimental checks (measurement of drift velocity) of electron thermalization. A further check (study of the slightly endothermic $e+\mathrm{D}$ I dissociative attachment) will be done soon.

$J$. H. Black: In studying the $e+\mathrm{C}_{14} \mathrm{H}_{10}$ reaction, do you measure the disappearance of electrons, or the anion itself?

$B$. Rowe: We have performed both kinds of measurements i.e. monitoring the electron density (measured by the Langmuir probe) decrease as a function of anthracene flow rate, and the appearance of the anthracene anions (detected by the quadrupole mass spectrometer). The rate coefficient obtained with both techniques are in agreement within the experimental uncertainty. 